\title{
Spinal Tuberculosis with Paraplegia in Pregnancy: a Case Report with Management of Spinal TB in Pregnancy
}

\author{
Sushruti Kaushal,' Santosh Kumar Dora,' Sunil Thakur ${ }^{2}$ \\ 'Department of Obstetrics \& Gynaecology, Vivekanand medical institute, palampur, Himachal Pradesh, ${ }^{2}$ Department of \\ anaesthesia and critical care, Vivekanand medical institute, palampur, Himachal Pradesh, India.
}

\section{ABSTRACT}

Spinal tuberculosis leading to paraplegia is uncommon in pregnancy and is a diagnostic and therapeutic challenge. We report a case of tubercular paraplegia presenting at 35 weeks of gestation. She was managed with Anti-tubercular drugs and did not require surgical intervention. Her neurological status improved and she was allowed to go in labour. She delivered a healthy term infant by cesarean. At three months follow-up, both mother and child are doing well.

Keywords: paraplegia; pregnancy; spinal tuberculosis.

\section{INTRODUCTION}

Tuberculosis is one of the oldest known infectious diseases that have seen resurgence in recent years. In 2012, there was estimated 8.6 million incident cases tuberculosis globally. Majority of the estimated cases in 2012 occurred in Asia (3.4 million) and Africa (2.3 million). ${ }^{1}$ Increased awareness among medical fraternity is required about rare presentations of tuberculosis in view of their rising incidence. Spinal tuberculosis leading to paraplegia is not common in pregnancy and is a diagnostic and therapeutic challenge We report a rare case of spinal tuberculosis presenting with paraplegia in pregnancy whose neurological deficit showed complete recovery with antitubercular chemotherapy.

\section{CASE REPORT}

A 27 yrs old second gravid gravida, with previous one spontaneous abortion, presented to our institute at 35 weeks of gestation with sudden onset, progressive weakness in bilateral lower limbs. Her medical, antenatal and past histories were unremarkable except a history of dull aching backache which had not been evaluated earlier. Her neurological deficit progressed in four days and she was unable to walk even with support. She had loss of touch and pain sensations in both lower limbs but her bladder and bowel functions were intact.

On neurological examination, tone \& power were normal in upper limbs bilaterally. In lower limbs, tone was normal \& power in proximal \& distal groups of muscle was $3 / 5 \& 4 / 5$ respectively on both sides. Deep tendon reflexes were increased and plantars were extensor. Pain sensation in bilateral lower limbs was diminished by $80-90 \%$; below T12 spinal level. Juxta Pyramidal System was impaired in bilateral lower limbs.

A MRI of dorsolumbar spine showed altered signal intensities in D9-10 and L3-4 vertebrae with wedge collapse of D10 vertebra. There was presence of abnormal prevertebral, paravertebral and epidural soft tissue causing thecal sac indentation and compression on cauda equine in the lumbar region. A provisional diagnosis of pott's spine involving D10-D9, L4-L3 vetebrae was made. An X ray of chest and lumbar spine was performed and was normal. . Patient was started on four drugs Anti Tubercular Therapy (ATT): isoniazide 300mg, rifampicin $450 \mathrm{mg}$, pyrizinamide $1500 \mathrm{mg}$ \& ethambutol $800 \mathrm{mg}$. In addition pyridoxine $50 \mathrm{mg}$ was started daily. Patient showed gradual improvement in motor power over the weeks. Patient was allowed to go into spontaneous labour at 37 weeks. She underwent an emergency cesarean section for fetal distress and delivered a male child weighing $2.76 \mathrm{~kg}$ with Apgar score

Correspondence: Dr. Sushruti Kaushal, Department of Obstetrics \& Gynaecology, Vivekan and medical institute, palampur, Himachal Pradesh, India. Phone Number: +91-9805657709, E-mail: sushrutikaushal@gmail.com. 
of 9/9. During shifting and caesarean care was taken for proper immobilization of the spine. Post operatively patient continued her ATT. She was discharged at day nine of surgery on intensive phase of ATT. Baby was evaluated for the evidence of congenital tuberculosis. Chest $X$ ray, gastric fluid aspiration for $A F B$ (Acid Fast Bacilli) \& USG abdomen done for the evidence of hepatosplenomegaly were normal. Neonate was started with prophylactic ATT with, isoniazide $5 \mathrm{mg} / \mathrm{kg}$, rifampicin $10 \mathrm{mg} / \mathrm{kg}$ body weight for three months. Baby didn't show any evidence of congenital tuberculosis at the age of three months and the patient was doing well with no motor deficits and minimal sensory deficits.

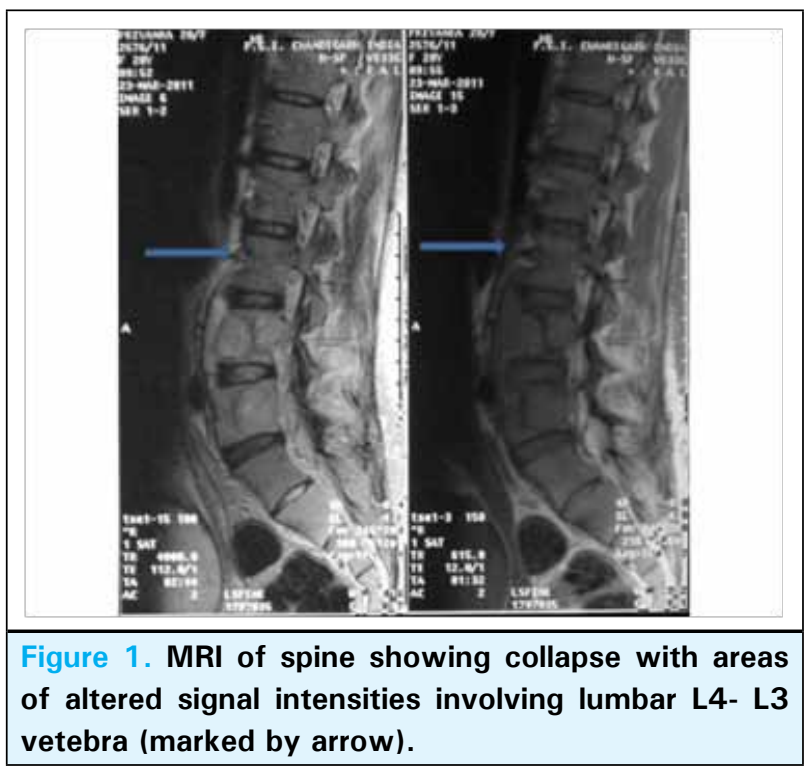

\section{DISCUSSION}

Tuberculosis of the spine with paraplegia in an unstable spine poses a special problem, the most difficult being performing spinal nursing in the presence of a rapidly enlarging gravid uterus in the third trimester. Dorsal followed by lumbar spine is the most common site to be involved. In our patient also there is involvement of these areas. Spinal tuberculosis occurs by secondary infection mainly by hematogenous or lymphatic route. More than $50 \%$ of cases of spinal tuberculosis have tubercular spondylitis which leads to pott's spine. Patient with this disease have symptoms of low back pain, vertebral deformity; psoas abscess etc. depending on site of involvement. ${ }^{2}$

\section{Management of spinal TB in pregnancy}

Tuberculosis of spine during pregnancy is rare. Pott's spine not complicated by neurological deficit or significant vertebral body destruction can be managed conservatively in the form of multidrug antitubercular chemotherapy. Pyridoxine supplementation and early mobilization in a suitable brace is appropriate. ${ }^{3}$ The safety of the first-line drugs for the management of active tuberculosis in pregnancy has been established, and therapy improves both maternal and neonatal outcomes. ${ }^{4}$ There is no malformation or teratogenicity identified in pregnant patients taking these drugs. Streptomycin should be avoided in pregnancy as it is ototoxic to the fetus. They can be given same dose of ATT as in non-pregnant patients. Directly observed therapy is recommended, especially if compliance to treatment is a concern. Patients on Isoniazid should be supplemented with $50 \mathrm{mg}$ of Pyridoxine,[5]. WHO recommends ATT for nine months but most experts prefer to continue ATT for 1-2 years.

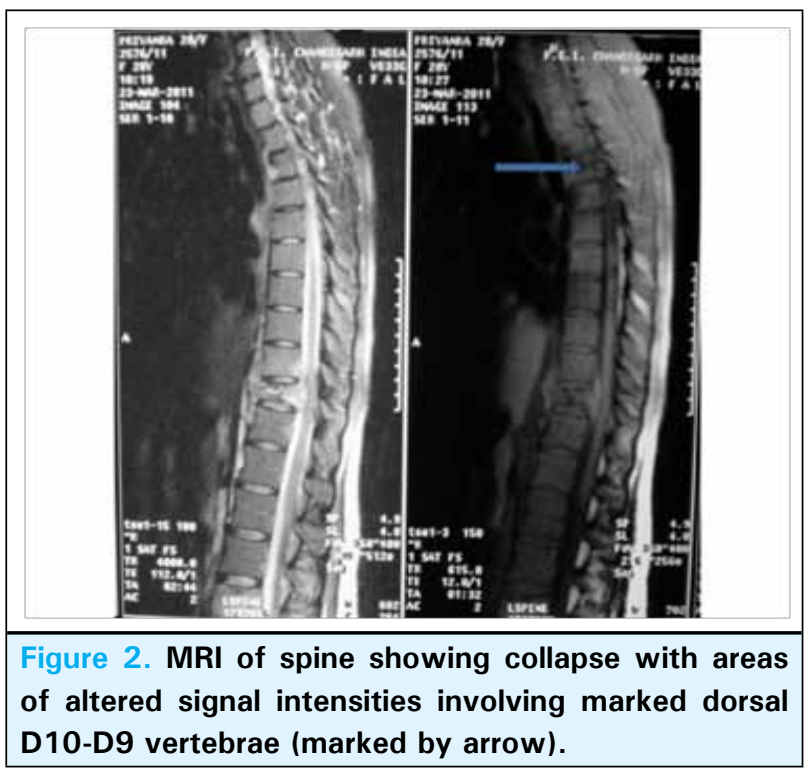

Pott's spine complicated by neurological deficits may require surgical decompression of the spine. Some experts advocate early surgical decompression in all while others prefer to operate after delivery. Surgery during pregnancy is difficult because of poor accessibility and poses risks of preterm labour and anaesthesia to developing fetus. While paraperesis or paraplegia can be effectively treated in some patients by chemotherapy as in ours, some require urgent cord decompression especially in patients with progressive neurological deficits or showing refractoriness to chemotherapy.

Patients with partial neurological deficit and short duration of symptoms have a higher chance of complete or near complete recovery. ${ }^{6,7}$

Pregnant women with paraplegia have increased incidence of urinary tract infection, decubitus ulcer, preterm labour \& autonomic hyperflexia. ${ }^{8}$ None of these were seen in our patient. Elective caesarean is not routinely indicated; decision to be made from case to case. Vaginal delivery is safe. ${ }^{9}$ There is no indication for early induction $\mathrm{i} / \mathrm{v} / \mathrm{o}$ spinal tuberculosis. 
Breast milk does not contain tuberculosis bacilli. Mother with active TB should be isolated from the child to reduce transmission rate irrespective of mode of breast feeding. All first line anti TB drugs is safe during breast feeding.

\section{REFERENCES}

1. Global tuberculosis report,WHO 2013.

2. Wurtz R, Quader Z, Simon D, Langer B. Cervical tuberculous vertebral osteomyelitis: case report and discussion of the literature. Clin Infect Dis 1993; 16: 806-808.

3. Tulli SM. Management and results. In: Tuberculosis of skeletal system, 3rd ed. New Delhi, India:Jaypee, 2004:265.

4. Efferen LS. Tuberculosis and pregnancy. Curr Opin Pulm Med,2007;13:205-1.

5. Clinical obstetrics and gynecology Volume 53, Number 2, 311-321,20108.
6. Kiran NS, Vaishya S, Kale SS, et al. Surgical results in patients with tuberculosis of the spine and severe lowerextremity motor deficits: a retrospective study of 48 patients. J Neurosurg Spine 2007;6:320-6.

7. Moon MS, Moon JL, Moon YW, et al. Pott's paraplegia in patients with severely deformed dorsal or dorsolumbar spines: treatment and prognosis. Spinal Cord 2003;41:164-1

8. Govender S, Moodley SC, Grootboom MJ. Tuberculosis paraplegia during pregnancy; a report of four cases. S Afr Med J 1989;75;190-2.

9. Jana N, Vasishta K, Saha S. C, Ghosh K.Obstetrical outcomes among women with extrapulmonary tuberculosis. (N Engl J Med 1999;341:645-9) 